\title{
Potencial toxicológico frente Artemia Salina em plantas condimentares comercializadas no município de Campina Grande-PB
}

\section{Toxicological potential Artemia Salina in front of condiments plants traded in Campina Grande-PB}

\author{
Emmanuel Moreira Pereira ${ }^{1}$; Manoel Tolentino Leite Filho ${ }^{1}$; Francisco de Assis Mendes ${ }^{1}$; Ana Nery Alves Martins ${ }^{2}$, Ana \\ Paula Trindade Rocha ${ }^{3}$
}

Resumo: O conhecimento do potencial toxicológico das plantas é um fator primordial para estipular o limite consumível. Objetivou-se nesse trabalho avaliar o potencial toxicológico de plantas condimentares frente Artemia salina, expostas às concentrações dos extratos das plantas erva-doce (Pimpinella anisum L.), pimenta malagueta (Capsicum frutescens), endro (Anethum graveolens L.) e alecrim (Rosmarinus officinalis L.). O trabalho foi desenvolvido no Laboratório de Engenharia de Alimentos - LEA pertencente ao Centro de Tecnologia e Recursos Naturais da Universidade Federal de Campina Grande-PB. A erva doce apresentou uma baixa toxicidade, atingindo uma DL50 de 428ppm do extrato. Já a pimenta malagueta exibiu um comportamento similar, atingindo o seu potencial toxicológico máximo na terceira diluição com DL50 716,1ppm. Para o endro, observou-se uma baixa taxa de mortalidade, consequentemente foi considerada não toxica, apresentando a DL50, com 2.624,5ppm. O alecrim apresentou baixos percentuais de mortalidade em náuplios de Artemia, sendo a DL50 com 3.720,6ppm, sendo considerado não toxico. No entanto necessita-se de estudos mais aprofundados para caracterizar a composição química das plantas estudadas e um teste in vivo para as que apresentaram baixa toxicidade.

Palavras-chaves: Erva doce, Pimenta Malagueta, Endro, Alecrim, toxicidade.

Abstract: Knowledge of the toxicological potential of plants is a key factor to stipulate the consumable limit. The objective of this study was to evaluate the toxicological potential of spice plants front Artemia salina exposed to concentrations of extracts of fennel plant (Pimpinella anisum L.), chili pepper (Capsicum frutescens), dill (Anethum graveolens L.) and rosemary ( Rosmarinus officinalis L.). The work was developed in Laboratório de Engenharia de Alimentos - LEA belonging to the Centro de Tecnologia e Recursos Naturais da Universidade Federal de Campina Grande-PB. The fennel has mild toxicity, reaching a LD50 of 428ppm extract. Have cayenne exhibited similar behavior, reaching its maximum in the third toxicological potential dilution with LD50 716,1ppm. For the dill, there was a low mortality rate, therefore was considered non-toxic, with the LD50 with 2.624,5ppm. Rosemary had low percentage of mortality in Artemia, with an LD50 with 3.720,6ppm and is considered non-toxic. However he will need further study to characterize the chemical composition of the studied plants and an in vivo test for presenting low toxicity.

Key words: Fennel, Chili Pepper, Dill, Rosemary, toxicity.

\footnotetext{
*Autor para correspondência

Recebido para publicação em 11/02/2015; aprovado em 15/03/2015

${ }^{1}$ Pós-graduando em Engenharia Agrícola - UFCG/CTRN - Universidade Federal de Campina Grande, e-mail: Emmanuel16mop@hotmail.com

${ }^{2}$ Graduada em licenciatura em química UEPB/CCT - Universidade Estadual da Paraíba

${ }^{3}$ Professora - UFCG/CTRN - Universidade Federal de Campina Grande
} 


\section{INTRODUÇÃO}

Muitas plantas são comercializadas a fim de incrementar o flavor de diversas iguarias culinárias, principalmente no nordeste do Brasil que é formado por uma mistura de raças e etnias, diversificando ainda mais a cultura culinária. No cenário medicinal são utilizadas diversas ervas como fitoterápicas, no entanto, pouco se sabe a respeito do potencial toxicológico dessas plantas. Por outro lado, a toxidez dos alimentos é um fator primordial para estabelecer o limite consumível do mesmo, no entanto, esse fator ainda é pouco visto diante de sua importância (Pereira et al., 2012). Diversas são as ervas aromáticas, condimentares e medicinais presentes no dia a dia dos nordestinos.

O amplo conhecimento sobre as plantas usadas pelas comunidades dar-se através da propagação do conhecimento tradicional compartilhado entre os membros da uma mesma família (Silva et al, 2014) ou seja um conhecimento sem fundamento cientifico fazendo se necessário um estudo mais aprofundado.

A erva-doce (Pimpinella anisum L.) também conhecida como anis é uma planta originária da costa mediterrânea, sendo usada como antiespasmódica, inibidora da fermentação intestinal e carminativa (Brunetton, 1991). Estudos recentes desvendaram novas e importantes atividades atribuídas à erva-doce. Os testes feitos por Boskabady e Ramazani-Assari (2001) constataram o efeito broncodilatador do óleo essencial e dos extratos etanólicos e aquosos da planta, apresentando forte atividade antioxidante e notável ação antibacteriana em bactérias Gram positivas e Gram negativas (Gülçin et al., 2003). Além disso, foi comprovado que o óleo essencial pode reduzir os efeitos causados pelo uso da morfina (Sahraei et al., 2002).

A pimenta malagueta (Capsicum frutescens) é um arbusto nativo das Américas, pertencente à família Solanaceae (Salomão, 2000). Esta família é conhecida por espécies de grande importância alimentícia e condimentar: batata (Solanum), cubiu, pimentas em geral, pimentão (Capsicum) e tomate (Lycopeisicon). A sua utilização para fins ornamentais (Datura), industriais como tabaco (Nicotiana), assim como medicinais e espécies venenosas que contenham alcalóides característicos como os dos gêneros Solanum, Capsicum e Lycopeisicon (Ribeiro et al.,1999).

Entre as pimentas, principalmente as malaguetas, são utilizadas para condimentar comidas e excitar o apetite, por causa da capsina, um alcalóide encontrado na semente, com característica acre, excitante e provoca localmente um estímulo rápido e energético (Braga, 1976).

O endro (Anethum graveolens L) é uma planta herbácea, de ciclo anual com altura média de até $1,20 \mathrm{~m}$, originária do mediterrâneo, utilizada para uso medicinal, condimentar e aromática, sendo caracterizada pelo tipo de folhas 3pinatisectas, flores amarelas dispostas em 4 a 8 umbelas e o fruto é um diaquênio com uma semente marrom (Couto, 2006).

O alecrim (Rosmarinus officinalis L.) é classificado pelo Council of Europe (1981) como um aromatizante natural, podendo ser adicionado aos alimentos. É uma das plantas medicinais mais conhecidas desde a Antiguidade, graças as suas propriedades medicinais, comestíveis e aromatizantes. Possuindo grande diversidade em sua composição química, como: Terpenóides (carnosol, ácido oleânico, ursólico, carnosílico); Flavonóides (apigenina, diosmetina, diosmina, genkwanina, luteolina, plantagina); ácidos fenólicos - cafeico, clorogênico, labiático, rosmarínico-colina, estigmasterol, taninos (Alonso 1998).

A Artemia salina é uma espécie de micro crustáceo da ordem Anostraca, muito utilizada como bioindicador em ensaios de laboratório, em função da sua facilidade de manipulação e do seu baixo custo econômico (Calow, 1993). O seu hábito alimentar é filtrador, se alimenta basicamente de bactérias, algas unicelulares, pequenos protozoários e detritos dissolvidos no meio. A filtração do alimento ocorre nos toracópodos encarregados de conduzir as partículas em direção ao trato digestivo, enquanto, a sua taxa de filtração diminui com o aumento da concentração de partículas, ficando estas acumuladas e interferindo o processo normal de seus batimentos. Outro efeito das altas concentrações é que podem passar diretamente pelo tubo digestivo sem sofrer digestão, tornando o indivíduo subnutrido (Souto, 1991). Estudos recentes comprovam a ação tóxica de várias substâncias naturais nestes pequenos animais (Rios, 1995; Nascimento et al., 2008).

Desta forma, este crustáceo tem sido utilizado em ensaios de toxicidade desenvolvidos para determinar a condição letal, de compostos bioativos em extratos vegetais (Meyer et al., 1982). De acordo com a Organização Mundial de Saúde (OMS), são consideradas tóxicas substâncias que apresentam valores de DL50 abaixo de 1000ppm em Artemia salina (Meyer et al., 1982). Normalmente os testes de toxicidade são elaborados com objetivo de avaliar ou prever os efeitos tóxicos em sistemas biológicos e dimensionar a toxicidade relativa das substâncias (Forbes e Forbes, 1994).

O objetivo deste trabalho foi avaliar o potencial toxicológico da erva-doce (Pimpinella anisum L.), pimenta malagueta (Capsicum frutescens), endro (Anethum graveolens L.) e alecrim (Rosmarinus officinalis L.), comercializados na feira livre de Campina Grande-PB, frente Artemia salina.

\section{MATERIAL E MÉTODOS}

A metodologia utilizada para os ensaios de toxicidade utilizando Artemia salina foi baseada em Meyer et al. (1982).

\section{Material Vegetal}

A erva-doce (Pimpinella anisum L.), pimenta malagueta (Capsicum frutescens), Endro (Anethum graveolens L.) e Alecrim (Rosmarinus officinalis L.) foram adquiridos em feira livre no mercado público de Campina Grande-PB. Em seguida, o material foi conduzido ao Laboratório de Engenharia de Alimentos - LEA pertencente à Centro de Tecnologia e Recursos Naturais da Universidade Federal Campina Grande-PB, onde foram preparados extratos e diluições a partir do material adquirido.

\section{Incubação da Artemia Salina}

Em um recipiente foram adicionados $500 \mathrm{~mL}$ de solução de água do mar artificial. O recipiente foi colocado dentro de uma incubadora iluminada por uma lâmpada fluorescente. No qual foram adicionados $0,2 \mathrm{mg}$ de cistos de Artemia salina, mantendo a água em agitação e aeração constante, com auxilio de um compressor de aeração para aquário. A incubação foi feita durante o período de 48 horas. 


\section{Exposição}

Após o período de incubação, os organismos-testes (náuplios de Artemia) foram expostos às amostras de interesse por 24 horas, utilizando-se tubos de ensaio graduados, cada um contendo 10 náuplios de Artemia salina, previamente selecionados. Os testes foram feitos em triplicata para cada concentração de cada composto. Além disso, cada droga foi testada, no mínimo, três vezes, totalizando um mínimo de 9 ensaios por concentração do composto.

Determinou-se a faixa de concentração a ser testada, buscando sempre a menor concentração em que se observasse $0 \%$ de mortalidade e a maior concentração em que se deflagrasse $100 \%$ de mortalidade. As demais concentrações foram distribuídas dentro desse limite (Veiga et al., 1989), de modo a obter a $\mathrm{DL}_{50}$ (dose letal para $50 \%$ da população) do composto testado.

\section{Contagem}

Após 24 horas de exposição, foi feita a contagem dos náuplios vivos e mortos, sendo considerados vivos todos aqueles que apresentassem qualquer tipo de movimento quando observados próximos a uma fonte luminosa. Só foram considerados válidos os testes nos quais o controle apresentou uma mortalidade igual ou inferior a $10 \%$ da população. Os resultados foram submetidos a tratamento estatístico utilizando o PROBIT, o qual forneceu os valores de $\mathrm{DL}_{50}$.

\section{RESULTADOS E DISCUSSÃO}

À medida que houve o acréscimo de concentração de erva doce (Pimpinella anisum L.), consequentemente elevouse a taxa de mortalidade (Figura 1), apresentando uma DL 50 de 428ppm (Tabela 1) sendo considerada possivelmente toxica, uma vez que a toxicidade de muitas substancias está ligada a sua composição química dentre eles as proteínas e compostos fenólicos. Em trabalhos desenvolvidos por Subehan et al., (2007) constatou-se diversos compostos fenólicos como: ácidos 3-cafeoilquínico, 4 -cafeoilquínico, 1,5-dicafeoilquínico, quercitrina, kaempferol-3-O-glicosídeo, miquelianina, narirituna, acacetin-3-O-rutinosideo, Kaempferol-3-O-rutinosideo, 6,8-C-diexosilapigenina, isoramnetin-3-O-rutinosídeo, escopoletina, bergapteno, psoraleno, 5-metoxipsoraleno e imperatorina em sua composição.

Para a pimenta malagueta (Capsicum frutescens) obteve comportamento similar da erva doceo, no entanto atingindo o seu potencial toxicológico máximo na terceira diluição (Figura 2), apresentando uma DL50 716,1ppm (Tabela 1) sendo considerada pouco toxica. O comportamento toxico da pimenta malagueta foi descrito em outros trabalhos, como no uso em forma de controle natural de pragas agrícolas em trabalhos desenvolvidos por Botti et al., (2014) utilizando estratos de pimenta no controle do ácaro-rajado constatou-se $97,4 \%$ de mortalidade. Esse potencial tóxico dar-se pelo fato que na sua composição encontra-se grande quantidade de compostos fenólicos e metabólicos secundários, tóxico em certas quantidade e a exposição à dada espécie. Em trabalhos descritos por Lee et al., (2005) destaca na composição da pimenta malagueta que dentre os composto fenólicos os flavonóides são em maior conteúdo encontrados nas pimentas destacando-se a quercetina e a luteolina, que estão presentes em formas conjugadas. Luteolina tem maior atividade antioxidante seguida pela capsaicina e pela quercetina.

\section{Erva Doce (Pimpinella anisum L.)}

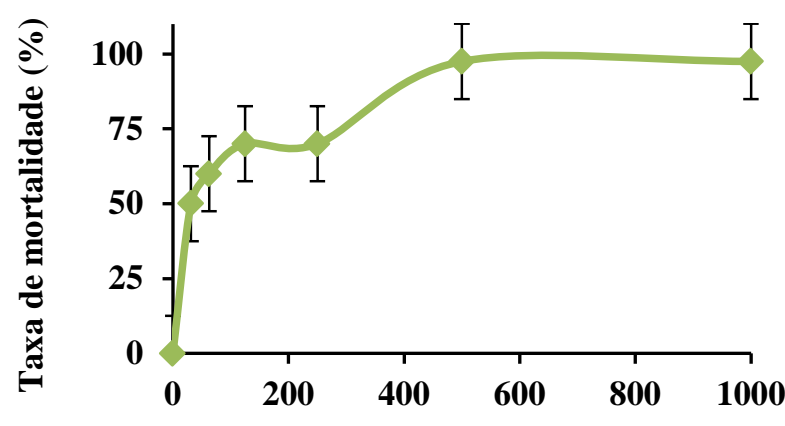

Consentração da amostra $\left(\mathrm{mg}^{-1}\right)$

Figura 1. Porcentagem de náuplios de Artemia salina mortos em relação ao aumento de concentração em Erva doce (Pimpinella anisum L.) comercializada no mercado publico em Campina Grande (—-). A barra vertical representa o desvio padrão da média. Campina Grande - PB, CTRN/UFCG, 2015.

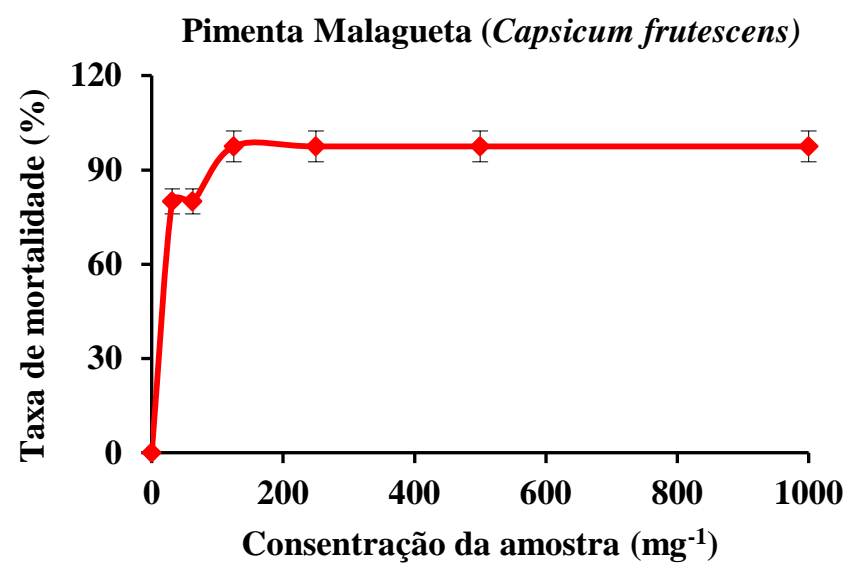

Figura 2. Porcentagem de náuplios de Artemia salina mortos em relação ao aumento de concentração em Pimenta malagueta (Capsicum frutescens) comercializada no mercado publico em Campina Grande (一-). A barra vertical representa o desvio padrão da média. Campina Grande - PB, CTRN/UFCG, 2015.

No entanto, para o endro (Anethum graveolens L) observou-se uma baixa taxa de mortalidade de náuplios de Artemia (Figura 3), apresentando uma DL50 2.624,5ppm (Tabela 1), consequentemente sendo considerada não toxica. Por essa característica o endro possui uma grande aplicabilidade por ser uma erva aromática e medicinal, presente em muitas regiões do Brasil, possuindo compostos benéficos ao tratamento e prevenção de doenças. Em trabalhos descritos por Singh et al., (2005) mostram que os extratos de endro (Anethum graveolens L) apresentam atividades antimicrobianas e antioxidantes. Sendo consumido sem restrição no uso de chás como um fitoterápico. 


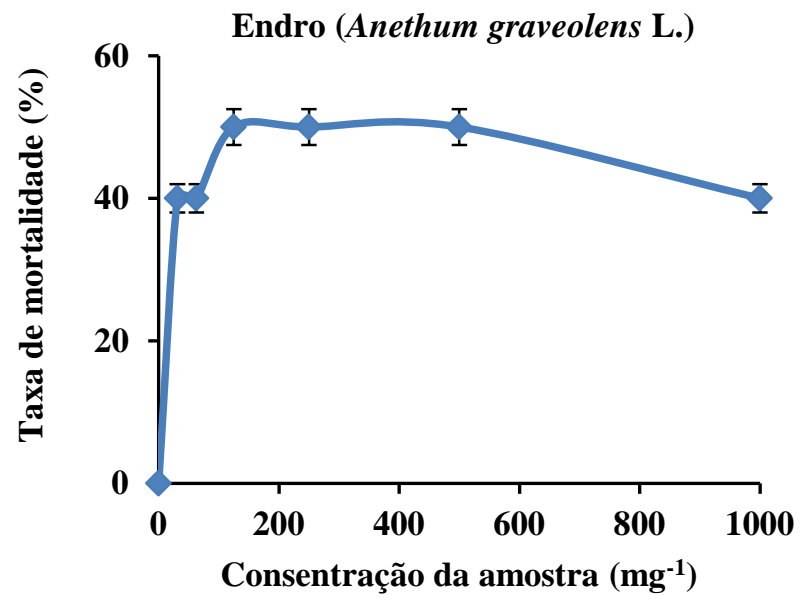

Figura 3. Porcentagem de náuplios de Artemia salina mortos em relação ao aumento de concentração em Endro (Anethum graveolens L.) comercializada no mercado publico em Campina Grande (- -). A barra vertical representa o desvio padrão da média. Campina Grande - PB, CTRN/UFCG, 2015.

$\mathrm{O}$ alecrim (Rosmarinus officinalis L.) apresentou um comportamento similar ao do endro, com baixos percentuais de mortalidade de náuplios de Artemia (Figura 1), apresentando uma DL50 de 3.1720,6ppm (Tabela 1), sendo considerada não toxica. A literatura apresenta poucos dados sobre a toxicidade do alecrim-pimenta (FARIAS et al., 2007). $\mathrm{O}$ alecrim apresenta em sua composição compostos benéficos não tóxicos, quando consumidos dentro dos limites. Em trabalhos por Almeida et al., (2010) com alecrim-pimenta identificam toxidade em testes in vivo com camundongos igual a $25 \mathrm{mg} \mathrm{kg}-1$. A toxicidade de varias plantas variam com as espécies em função sua composição química.

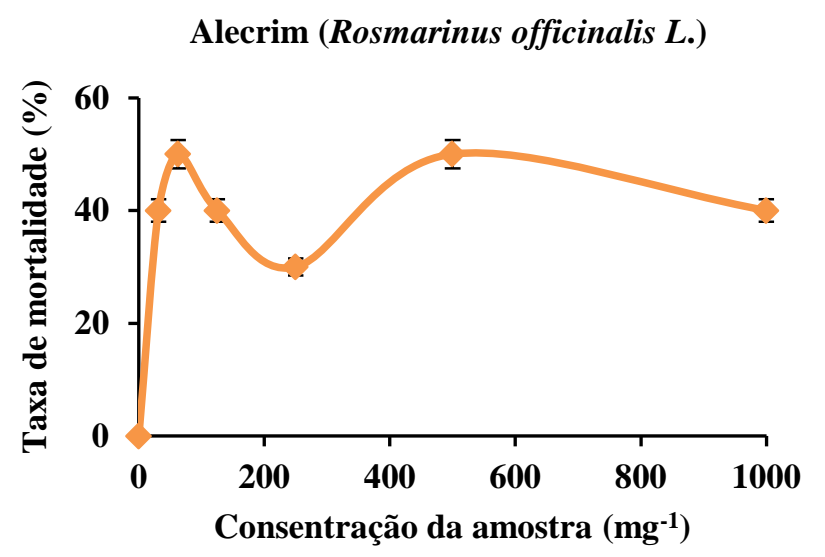

Figura 4. Porcentagem de náuplios de Artemia salina mortos em relação ao aumento de concentração em Alecrim (Rosmarinus officinalis L.) comercializada no mercado publico em Campina Grande $(-\downarrow)$. A barra vertical representa o desvio padrão da média. Campina Grande - PB, CTRN/UFCG, 2015.

Tabela 1: Valores de Dose letal (DL50) e limites superiores e inferiores para Erva doce, Pimenta malagueta, Endro e Alecrim.

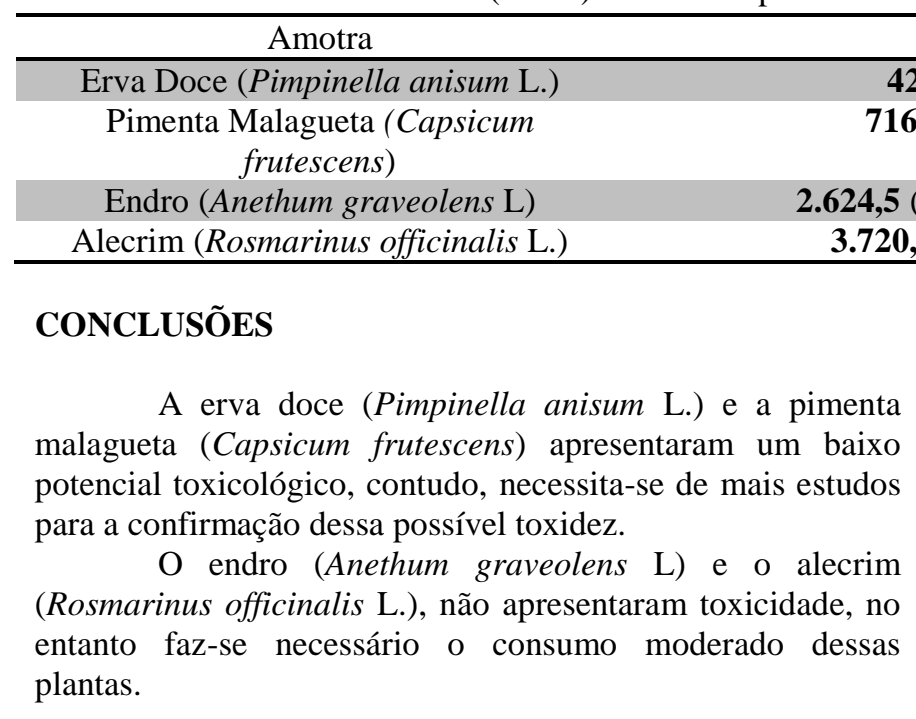

\section{REFERÊNCIAS BIBLIOGRÁFICAS}

ALMEIDA, A. C., SOBRINHO, E. M., PINHO, L. D., SILVA, P. N., MARTINS, E. R., \& DUARTE, E. R. Toxicidade aguda dos extratos hidroalcoólicos das folhas de alecrim-pimenta, aroeira e barbatimão e do farelo da casca de pequi administrados por via intraperitoneal. Ciência Rural, 40(1), 2010.

ALONSO, J. R. Tratado de fitomedicina - bases clínicas e farmacológicas, editora Isis . $1998 . \quad$ Disponível em: http://www1.londrina.pr.gov.br/dados/images/stories/Sto
428 (limite inferior 6,6533- limite superior 83,5)

716,1 (limite inferior 3,6584- limite superior 830,6)

24,5 (limite inferior 6.130,7548 - limite superior 11.379,7)

.720,6 (limite inferior 2.485,9 - limite superior 8.327,2)

rage/sec_saude/fitoterapia/publicacoes/alecrim2.pdf; acessado em: 14/10/2014.

BOTTI, J. M. C., HOLTZ, A. M., PIRES, A. A., \& FONTES, P. S. F. Potencial de uso de extratos de mamona, pimenta e pinhão manso sobre o manejo do ácaro rajado do mamão. VII Jornada de iniciação cientifica, desenvolvimento em tecnologia e inovação dos Ifs; In anais; 2014.

BOSKABADY, M.H., RAMAZANI-ASSARI, M. Relaxant effect of Pimpinella anisum on isolated guinea pig tracheal chains and its possible mechanism(s). Journal of Ethnopharmacology, v.74, p.83-8, 2001.

BRAGA, R. Plantas do Nordeste, especialmente do Ceará. 3. ed., Fortaleza: Escola Superior de Agricultura de Mossoró, 1976, 234 p.

BRUNETON, J. Elementos de fitoquímica e farmacognosia. Zaragoza: Acriba, 1991. 594p. 
CALOW, P. Marine and estuarine invertebrate toxicity tests. In: HOFFMAN, D. et al. Handbook in cytotoxicology. Oxford: Blackwell Scientific Publication, 1993. v. 1. p. $1-5$.

COUTO, M. E.; Coleção de plantas medicinais aromáticas e condimentares. --Pelotas: Embrapa Clima Temperado-(Embrapa Clima Temperado. Documentos, 157). ISSN 1806-9193. 2006. 91 p.

FARIAS, E.M.F.G. et al. Avaliação da toxicidade aguda do extrato metanólico de folhas de Lippia sidoides Cham. (Verbenaceae) In. CONGRESSO BRASILEIRO DE QUÍMICA, 2007, Natal, RN. Anais... Natal: Sociedade Brasileira de Química. 2007. Disponível em: <http://www.abq.org.br/cbq/2007/trabalhos/7/7-600750.htm>. Acesso em: 01 Outubro. 2014.

FORBES, V. E.; FORBES, T. L. Ecotoxicology in theory and practice. Londres: Chapman and Hall, 1994. 247 p.

GÜLÇIN, I., OKTAY, M., KÜFREVIOGLU, O.I. Screening of antoxidant and antimicrobial activities of anise (Pimpinella anisum L.) seed extracts. Food Chemistry, v.83, p.371-82, 2003.

LEE, J. J., CROSBY, K. M., PIKE, L. M., YOO, K. S., \& LESKOVAR, D. I. Impact of genetic and environmental variation on development of flavonoids and carotenoids in pepper (Capsicum spp.). Scientia Horticulturae, v.106, p.341-352, 2005.

MEYER, B. N., FERRIGNI, N. R., PUTNAN, J. E., JACOBSEN, L. B., NICHOLS, D. E., Mcl. AUGHLIN, J. Brine shrimp: A convenient general bioassay for active plant constituents. Journal of Medical Plant Research, v. 45, n.1, p. 31-34, 1982.

NASCIMENTO, J.E.; MELO, A.F.M.; LIMA E SILVA, T.C.; VERAS FILHO, J.; SANTOS, E.M.; ALBUQUERQUE, U.P.; AMORIM, E.L.C. Estudo fitoquímico e bioensaio toxicológico frente a larvas de Artemia salina Leach. de três espécies medicinais do gênero Phyllanthus (Phyllanthaceae). Journal of Basic and Applied Pharmaceutical Sciences, v. 29, n.2, p. 143$148,2008$.

PEREIRA EM; COSTA, RTRV; COSTA FB; FERREIRA AA; ARAÚJO HG; ARAÚJO, AS; CAVALCANTI, MT 2012. Avaliação microbiológica e toxicológica de broto de palma inteiro e minimamente processado. In: CONGRESSO BRASILEIRO DE OLERICULTURA, 52. Anais... Salvador: ABH.2012.

RIBEIRO, J. E. L. S.; HOPKINS, M.; VICENTINI, A.; SOTHERS, C. A .; COSTA, M. A. S.; BRITO, J. M.;
SOUZA, M. A. D.; MARTINS, L. H. P.; LOHMANN, L. G.; ASSUNÇÃO, P. A. C. L.; PEREIRA, E. C.; SILVA, E. C.; SILVA, C. F.; MESQUITA, M. R. e PROCÓPIO, L. C. Flora da Reserva Ducke. Manaus: Inpa-DFID, 1999, $285 \mathrm{p}$.

RIOS, F. J. B. Digestibilidade in vitro e toxicidade de lectinas vegetais para náuplios de Artemia sp.1995. Dissertação (Mestrado em Bioquímica) - Universidade Federal do Ceará, Fortaleza-Ce.

SAHRAEI, H., GHOSHOONIA, H., SALIMIB, S.H. et al. The effects of fruit essential oil of the Pimpinella anisum on acquisition and expression of morphine induced conditioned place preference in mice. Journal of Ethnopharmacology, v.80, p.43-7, 2002.

SALOMÃO, J. A. F. Globo Rural Responde, ed. 182Dez/2000. Disponível em: http:// www. globorural.globo.com/barra.asp?d=/ edic/182/sumario.htm. Acesso em: 20 de Setembro de 2014.

SILVA, S.; ANSELMO, M.G.V.; DANTAS, W.M.; ROSA, J.H.; NUNES, E.N.; SOARES, J. P.; ALVES, C.A.B. Conhecimento e uso de plantas medicinais em uma comunidade rural no município de Cuitegi, Paraíba, Nordeste do Brasil, Gaia Scientia, v 8, n 1, p 248-265, 2014.

SINGH,G .; MAURYA, S.; LAMPASONA, M.; CATALAN, C. Chemical Constituents, Antimicrobial Investigations, and Antioxidative Potentials os Anethum graveoles L. Essential Oil and Acetone Extract: Part 52. Jornal os Food Science, v.70, n. 4, p. 208-215. 2005.

SOUTO, F. J. B. Influencias de parâmetros ambientais sobre Artemia sp (Branchiopoda: Artemiidae) em uma salina artesanal do estado do Rio Grande do Norte. Curso de Mestrado em Ciências Biológicas - Zoologia, Universidade Federal da Paraíba, 19 p. Notas de aula. 1991.

SUBEHAN. ZAIDI, S. F. H.; KADOTA, S.; TEZUKA, Y. Inhibition on Human Liver Cytochrome P450 3A4 by Constituents of Fennel (Foeniculum vulgare): Identification and Characterization of a MachanismBased Inactivator. Journal of Agricultural and Food Chemistry, v. 55, n. 25, p. 10162-10167. 2007.

VEIGA, L. F.; VITAL, N .A.; PORTELA, M. R.; OLIVEIRA, F. F. Avaliação de faixa de sensibilidade de Artemia salina ao Lauril Sulfato de Sódio. Rio de Janeiro. PETROBRÁS/CENPES/SUPESQ/DITER, 64p. il.. 1989. 